\title{
Líneas para el desarrollo de proyectos con Wikipedia y Wikimedia Commons en museos y bibliotecas.
}

\author{
Rubén Ojeda. \\ Coordinador de Programas, Wikimedia España. \\ rubenojeda@wikimedia.es \\ Jesús Tramullas \\ Depto. Ciencias de la Documentación, Univ. de Zaragoza. \\ tramullas@unizar.es
}

\begin{abstract}
Resumen:El movimiento OpenGLAM tiene como objetivo la publicación, bajo licencias libres, de material cultural digitalizado. Las instituciones culturales (museos, bibliotecas y archivos) son actores clave para el acceso y la difusión del patrimonio cultural. Este texto explica los fundamentos, planificación y actividades llevadas a cabo en el proyecto GLAMing Madrid, en el que Wikimedia España participó, junto al Museo Arqueológico Nacional, el Museo del Romanticismo y el Museo del Traje. Finalmente, se indican los resultados obtenidos y las buenas prácticas identificadas.
\end{abstract}

Palabras clave: GLAM, OpenGLAM, GLAMing Madrid, instituciones culturales, wikipedista residente, editatones

Abstract: The objective of the OpenGLAM movement is the publication, under free licenses, of digitized cultural material. Cultural institutions (museums, libraries and archives) are key actors for the access and dissemination of cultural heritage. This text explains the foundations, planning and activities carried out in the GLAMing Madrid project, in which Wikimedia Spain participated, together with the National Archaeological Museum, the Museum of Romanticism and the Costume Museum. Finally, the results obtained and the good practices identified are indicated.

Keywords: GLAM, OpenGLAM, GLAMing Madrid, wikipedian in residence, editathons

\section{Wikipedia y las bibliotecas.}

Los objetivos de Wikipedia y de las bibliotecas coinciden en pretender poner al alcance de la sociedad el acceso a la cultura y a la ciencia, de una forma abierta, y en desarrollar los procesos de competencia informacional de sus usuarios ${ }^{1}$. El desempeño del sistema bibliotecario en el acceso y divulgación ha sido identificado como un factor clave por el propio movimiento wikipedista, el cual ha incluido en sus proyectos diversas iniciativas que integran el ámbito bibliotecario, tanto a nivel de grupos de usuarios, como de recursos de información, como de actividades de edición y liberación de contenidos. The Wikipedia Library ${ }^{2}$, Wikipedia Library User Group ${ }^{3}$, 1LiblRef ${ }^{4}$, $G L A M-W I K I^{5}$ son ejemplos de cómo abordar esta integración desde la comunidad wikipedista. A ellos cabría citar los numerosos eventos que han tenido como marco instituciones bibliotecarias.

1 CATALANI, Luigi. Biblioteche e Wikimedia: strategie comuni per l'accesso aperto alla conoscenza e la costruzione collaborativa del sapere libero. JLIS.it, [en línea], 2017, vol. 8, núm. 3 [consulta: 22 noviembre 2017]. Disponible en: doi:10.4403/jlis.it-12413

2 Véase https://meta.wikimedia.org/wiki/The_Wikipedia_Library

3 Véase https://meta.wikimedia.org/wiki/Wikipedia_Library_User_Group

4 Véanse la definición y las características del proyecto 1Lib1Ref en https://meta.wikimedia.org/wiki/The_Wikipedia_Library/1Lib1Ref/es

5 Véase https://outreach.wikimedia.org/wiki/GLAM 
Con motivo de la conferencia celebrada por la IFLA en 2016 en Columbus (Ohio), se presentaron sendos documentos trabajo, titulados Opportunities for Public Libraries and Wikipedia ${ }^{6}$ y

Opportunities for Academic and Research Libraries and Wikipedia ${ }^{7}$ respectivamente, en los que dos paneles de expertos revisaban las posibilidades que se abren a las bibliotecas, tanto públicas como pertenecientes a entidades dedicadas a la investigación, y las diferentes vías en las que ambas partes podían integrarse satisfactoriamente, y beneficiarse mutuamente para el cumplimiento de sus metas y objetivos.

Las vías de esta colaboración pueden incluir la realización de actividades de formación de los profesionales y de los usuarios, atendiendo a competencias de organización, redacción y referencia de información, las competencias informacionales, la integración con linked open data, la potenciación de los catálogos y colecciones bibliotecarias, el desarrollo del acceso abierto, la creación de comunidades de aprendizaje y la integración en procesos de enseñanza/aprendizaje ${ }^{8}$. Además de todo ello, hay que tener en cuenta que las bibliotecas son consideradas como una parte activa del movimiento GLAM (Galleries, Libraries, Archives and Museums), en lo concerniente a los procesos de digitalización y liberación de contenidos culturales ${ }^{9}$. Aunque generalmente se asocian las actividades GLAM a instituciones museísticas, las bibliotecas, cualquiera que sea su tipo, se configuran como socios potenciales y activos de este tipo de proyectos. Si se trata de bibliotecas de museos, resulta evidente que la capacidad y las posibilidades para la realización de proyectos colaborativos sobre el patrimonio cultural aumentan de forma exponencial.

\section{EI movimiento OpenGLAM.}

Los proyectos GLAM se asocian a la liberación de materiales culturales digitalizados, que se ponen a disposición de todos los usuarios, bajo licencias libres, sin que existan cortapisas para su utilización, sea comercial o no. Este movimiento se imbrica en el más amplio marco del movimiento de acceso abierto, que progresivamente ha ido ampliando sus objetivos, hasta incluir elementos y colecciones de patrimonio cultural, que dadas las características del acceso, son resultantes de procesos de digitalización ${ }^{10}$. Instituciones como Library of Congress, British Library, British Museum, Koninklijke Bibliotheek, OCLC, METS... han llevado a cabo, o participan actualmente, en diferentes tipos de actividades cuya finalidad es poner a disposición de los ciudadanos diversos tipos de contenidos en la red, de forma que se pueden disfrutar y aprovechar libremente. Los materiales digitalizados se publican con licencias libres, lo que hace posible su utilización sin cortapisas en cualquier contexto: Creative Commons de Dominio Público (CCO), Atribución (CC-BY) y Atribución-Compartir Igual (CC-BY-SA), Licencia de Documentación Libre de GNU, Free Art License y licencias Open Data Commons. Esta aproximación facilita su uso en actividades educativas, creativas y económicas, ofreciendo numerosos beneficios a la sociedad.

6 FENOLL, Carme, et alii. Opportunities for Public Libraries and Wikipedia. A Discussion Paper (Public Draft) Presented at the IFLA World Library and Information Congress 2016 in Columbus, Ohio. [en línea]. 2016. [consulta: 8 octubre 2017]. Disponible en: https://2016.ifla.org/wp-content/uploads/sites/2/2016/08/112IFLAWikipediaandPublicLibrariesDiscussionDRAFT.pdf

7 BARTHOLMEI, Stephan, et alii. Opportunities for Academic and Research Libraries and Wikipedia. A Discussion Paper (Public Draft) Presented at the IFLA World Library and Information Congress 2016 in Columbus, Ohio. [en línea]. 2016. [consulta: 8 octubre 2017]. Disponible en: https://2016.ifla.org/wp-content/uploads/sites/2/2016/08/112IFLAWikipediaAcademicandResearchLibrariesDiscussioDRAFT.pdf

8 AYERS, Phoebe. At scale, at large: Wikipedia \& Libraries. [en línea]. 2016. [consulta: 20 diciembre 2017]. Disponible en: https://medium.com/@phoebeayers/at-scale-at-large-wikipedia-libraries-3e90a9618398

9 SAORÍN, Tomás. Iniciativas GLAM-Wiki: Wikipedia como oportunidad para instituciones culturales. Anuario ThinkEPI, [en línea], 2013. vol. 7, págs. 78-85. [consulta: 14 octubre 2017]. Disponible en: https://recyt.fecyt.es/index.php/ThinkEPI/article/view/30335

10 KAPSALIS, Effie. The Impact of Open Access on Galleries, Libraries, Museums, \& Archives. [en línea]. Smithsonian Institution, 2016, [consulta: 3 octubre 2017]. Disponible en: http://siarchives.si.edu/sites/default/files/pdfs/2016_03_10_OpenCollections_Public.pdf 
Europeana ha establecido una línea directa de colaboración con diferentes grupos nacionales de editores de Wikipedia para estas acciones, indicándolo además en sus documentos de estrategia para 2020 .

La preocupación de la Unión Europea por esta cuestión se ha visto reflejada en la Recomendación de la Comisión de 27 de octubre de 2011 sobre la digitalización y accesibilidad en línea del material cultural y la conservación digital, la cual, entre otras cuestiones, señala específicamente lo siguiente sobre el material digitalizado por instituciones públicas ${ }^{11}$ :

“5a) garantizando que el material de dominio público siga perteneciendo al dominio público una vez digitalizado;

5b) promoviendo un acceso lo más amplio posible al material digitalizado de dominio público y a la reutilización de este material para fines comerciales y no comerciales; 5c) adoptando medidas para limitar el uso de marcas intrusivas u otras medidas de protección visual que dificulten la utilización del material digitalizado de dominio público.

7c) garantizando el acceso a todas sus obras maestras de dominio público a través de Europeana antes de 2015;"

Posteriormente, la Unión Europea puso en marcha y financió hasta 2016 la iniciativa OpenGLAM, ${ }^{12}$ coordinada por el Open Knowledge Project, cuya finalidad era construir una cultura global sobre el procomún, y que desarrolló actividades dinamizadoras sobre la liberación de contenidos culturales y su reutilización en diferentes campos. La Unión Europea también financió, entre 2014 y 2017, el proyecto Europeana $\mathrm{Space}^{13}$, para el desarrollo de nuevas oportunidades para el crecimiento económico y social en el sector de las industrias creativas, a través de la reutilización de contenido cultura digitalizado.

Como actor del movimiento OpenGLAM, y parte activa del movimiento Wikimedia, Wikimedia España ${ }^{14}$ desarrolla trabajo voluntario colaborando con instituciones y usuarios de Wikipedia y otros proyectos Wikimedia. Dentro de sus actividades también lleva a cabo colaboraciones con museos, bibliotecas y archivos para la organización de eventos de edición y en el desarrollo de procesos de liberación y difusión de contenidos ${ }^{15}$. Además de estas actuaciones, debe destacarse su actividad en el ámbito de los concursos fotográficos. Wiki Loves Monuments ${ }^{16}$ se ha convertido en el mayor concurso mundial de fotografía de patrimonio cultural ${ }^{17}$. Con la misma aproximación se han creado y se gestionan Wiki Loves Folk ${ }^{18}$, para la fotografía de fiestas de interés turístico, y Wiki Loves $\operatorname{Earth}^{19}$, para fotografía sobre espacios naturales protegidos.

11 COMISIÓN EUROPEA. Recomendación de la Comisión de 27 de octubre de 2011 sobre la digitalización y accesibilidad en línea del material cultural y la conservación digital. [en línea]. Diario Oficial de la Unión Europea, 2011, L283/30-45 [consulta: 6 septiembre 2017]. Disponible en: http://eur-lex.europa.eu/LexUriServ/LexUriServ.do?uri=OJ:L:2011:283:0039:0045:ES:PDF

12 Véase https://openglam.org/

13 Véase http://www.europeana-space.eu/

14 Wikimedia España es una Asociación sin ánimo de lucro, inscrita en el Registro Nacional de Asociaciones del Ministerio del Interior desde marzo de 2011. Fue aprobada como capítulo estatal español por la Wikimedia Foundation en febrero de 2011.

15 OJEDA, Rubén. y HERNÁNDEZ-CAZORLA, Iván. Wikimedia España y la difusión del patrimonio cultural. Revista PH [en línea]. 2017, vol. 91, págs. 36-37, [consulta: 2017-10-10]. Disponible en: http://www.iaph.es/revistaph/index.php/revistaph/article/view/3882

16 Véase https://www.wikilm.es/

17 RODRÍGUEZ-POSADA, Emilio José et alii. Wiki Loves Monuments 2011: experiencia en España y reflexiones desde la difusión del patrimonio cultural.Digithum, [en línea], 2012, n. 14, [consulta: 12 noviembre 2017] Disponible en: http://doi.org/10.7238/d.v0i14.1472

18 Véase https://www.wikilov.es/es/Wiki_Loves_Folk

19 Véase https://www.wikilovesearth.es/ 


\section{El Proyecto GLAMing Madrid.}

Wikimedia España, en el marco de sus actividades habituales, había mantenido contactos con diferentes museos entre 2013 y 2014. A comienzos de 2015 se entró en contacto con varios museos estatales, que mostraron interés en colaborar en la realización de actividades conjuntas que facilitasen la difusión de sus colecciones. Una vez analizado el contexto de trabajo y las perspectivas de los museos que iban a tomar parte, se consideró que era necesario llevar a cabo el proyecto a través de un wikipedista residente. La figura del wikipedista residente ${ }^{20}$, ampliamente utilizada en proyectos que envuelven la integración en Wikipedia y Wikimedia Commons de material digitalizado procedente de instituciones culturales y de la memoria, pretende que las instituciones integren en su trabajo diario un especialista externo, proveniente el mundo de los proyectos Wikimedia. La misión de este especialista es desarrollar proyectos que permitan formar al personal de la institución, y planificar acciones que pongan en valor las colecciones de la institución, con especial atención a la creación de contenidos y a la publicación de material digitalizado. Los resultados de estas acciones deben difundirse con licencias que hagan posible un uso libre, de manera que se potencien la misión de las instituciones implicadas y se facilite la difusión del patrimonio cultural.

Las entidades que decidieron participar en la iniciativa fueron el Museo del Romanticismo, el Museo del Traje y el Museo Arqueológico Nacional. Dadas las características del proyecto que se pretendía llevar a cabo, era necesario disponer de financiación, lo que resultaba casi imposible en ese momento, dadas las características y estructura de Wikimedia España, por un lado, y los recortes sufridos por las instituciones a consecuencia de la crisis económica. Por estos motivos, y aprovechando las opciones de financiación de acciones que ofrece la Wikimedia Foundation, se optó por preparar, redactar y solicitar un Project and Event Grant (PEG), que fue presentado a finales de enero de 2015, y aprobado en marzo del mismo año ${ }^{21}$. Posteriormente, para dar cobertura legal al trabajo en los citados museos, se redactó y firmó un convenio con el Ministerio de Educación, Cultura y Deporte, en junio de 2015, que incluía un anexo con el conjunto de museos estatales en los cuales sería de aplicación el convenio.

El proyecto GLAMing Madrid se desarrolló entre el 7 de septiembre de 2015 y el 7 de enero de $2016^{22}$. Una vez resueltos los aspectos de financiación y administrativos, se abordó con los museos la estructura y organización del trabajo a desarrollar. Con la finalidad de equilibrar y extender en el tiempo las actividades, se acordó acudir a los tres museos cada semana, alternando los días, y respetando el horario de cada institución. En cada uno de ellos se trabajó haciendo uso de los espacios que habían sido dispuestos a tal fin.

La planificación de actividades contempló la ejecución de un conjunto de acciones que hiciesen posible, en primer lugar, la integración de los diferentes museos en el procesos de trabajo y en la comunidad de Wikipedia; en segundo, la dinamización de los museos mediante la celebración de actividades para sus usuarios, y, en tercer lugar, la introducción y participación de los museos en los procesos de liberación de contenidos en proyectos GLAM. La tipología de las actividades que se llevaron a cabo responde a un modelo clásico de desarrollo de proyectos GLAM, similar a los que se han llevado a cabo en otras instituciones museísticas:

- Talleres de formación: acciones destinadas a la formación básica de los usuarios en la estructura, el funcionamiento, la comunidad y las dinámicas básicas de edición y creación de

20 ORLOWITZ, Jake y EARLEY, Patrick, Librarypedia: The future of Libraries and Wikipedia. [en línea] TheDigitalShift, [consulta 2 de mayo de 2017]. Disponible en: http://www.thedigitalshift.com/2014/01/discovery/librarypedia-future-libraries-wikipedia/

21 Véase https://meta.wikimedia.org/wiki/Grants:PEG/WM_ES/GLAMing_Madrid

22 OJEDA, Rubén y WIKIMEDIA ESPAÑA. Proyecto GLAMing Madrid. [en línea]. 2016. [consulta: 7 septiembre 2017]. Disponible en: https://commons.wikimedia.org/wiki/File:Memoria_GLAMing_Madrid-es.pdf 
contenidos en Wikipedia y Wikimedia Commons (véase tabla 1). Los talleres se dirigieron a dos tipos de usuarios diferenciados:

- Talleres para formación del personal del museo: destinados a formar al personal de los museos participantes. La secuencia de formación empezaba con una charla introductoria a Wikipedia, que era seguida, en un momento posterior, por uno, o varios, talleres de edición básica de contenidos (estructura de artículos, páginas de discusión, técnicas de edición, etc...)

- Talleres públicos: se llevaron a cabo en cada uno de los museos participantes, y estaban destinados a los usuarios de los mismos. Su planificación se llevó a cabo de forma conjunta con cada museo, ajustando el número de talleres, los días de celebración, la difusión y el contenido temático de cada uno de ellos. Una vez definida la temática de cada taller, las bibliotecas de los museos eran las encargadas de preparar las herramientas bibliográficas necesarias para los usuarios o editores, y ponerlas a su disposición para consulta en el espacio en el que se llevaba a cabo el taller. En el Museo Arqueológico Nacional y en Museo del Traje los talleres para el público se llevaron a cabo, precisamente, en los espacios de las bibliotecas correspondientes. Las aportaciones de los participantes fueron controladas y cuantificadas usando la herramienta Wikimetrics.

\begin{tabular}{|l|c|c|}
\hline \multicolumn{3}{|l}{ Talleres de edición para usuarios de los museos } \\
\hline Museo & Talleres realizados & Artículos editados \\
\hline Museo Arqueológico Nacional & 7 & 66 \\
\hline Museo del Romanticismo & 5 & 19 \\
\hline Museo del Traje & 6 & 29 \\
\hline
\end{tabular}

Talleres de introducción a Wikipedia para personal del museo

\begin{tabular}{|l|c|c|}
\hline Museo & Talleres realizados & Participantes \\
\hline Museo Arqueológico Nacional & 3 & 22 \\
\hline Museo del Romanticismo & 2 & 9 \\
\hline Museo del Traje & 1 & 6 \\
\hline
\end{tabular}

Tabla 1. Datos sobre los talleres desarrollados en el proyecto GLAMing Madrid.

- Trieditatón: se trató de una actividad presencial abierta al público, que contaba también con la posibilidad de participar de forma no presencial, de edición temática de contenidos de los tres museos ${ }^{23}$. Se celebró en la biblioteca del Museo Arqueológico Nacional el 17 de octubre de 2015, en sesión continua entre las 11 y las 18 horas. Contó con catorce asistentes, que crearon seis nuevos artículos e hicieron un total de 196 ediciones.

- Conciertos de música clásica: El Museo del Romanticismo programa interpretaciones de música clásica. Se planteó la posibilidad de que los intérpretes colaborasen en el proyecto GLAMing Madrid permitiendo la grabación de sus actuaciones. Se grabaron exitosamente dos actuaciones en noviembre de 2015, realizándose las mismas en privado para evitar los problemas de ruido de fondo de las grabaciones con público. Los audios, de obras de Albéniz, Ravel, Debussy y Chopin, fueron aportados a Wikimedia Commons, y posteriormente facilitaron la creación de la herramienta Wikiradio. 


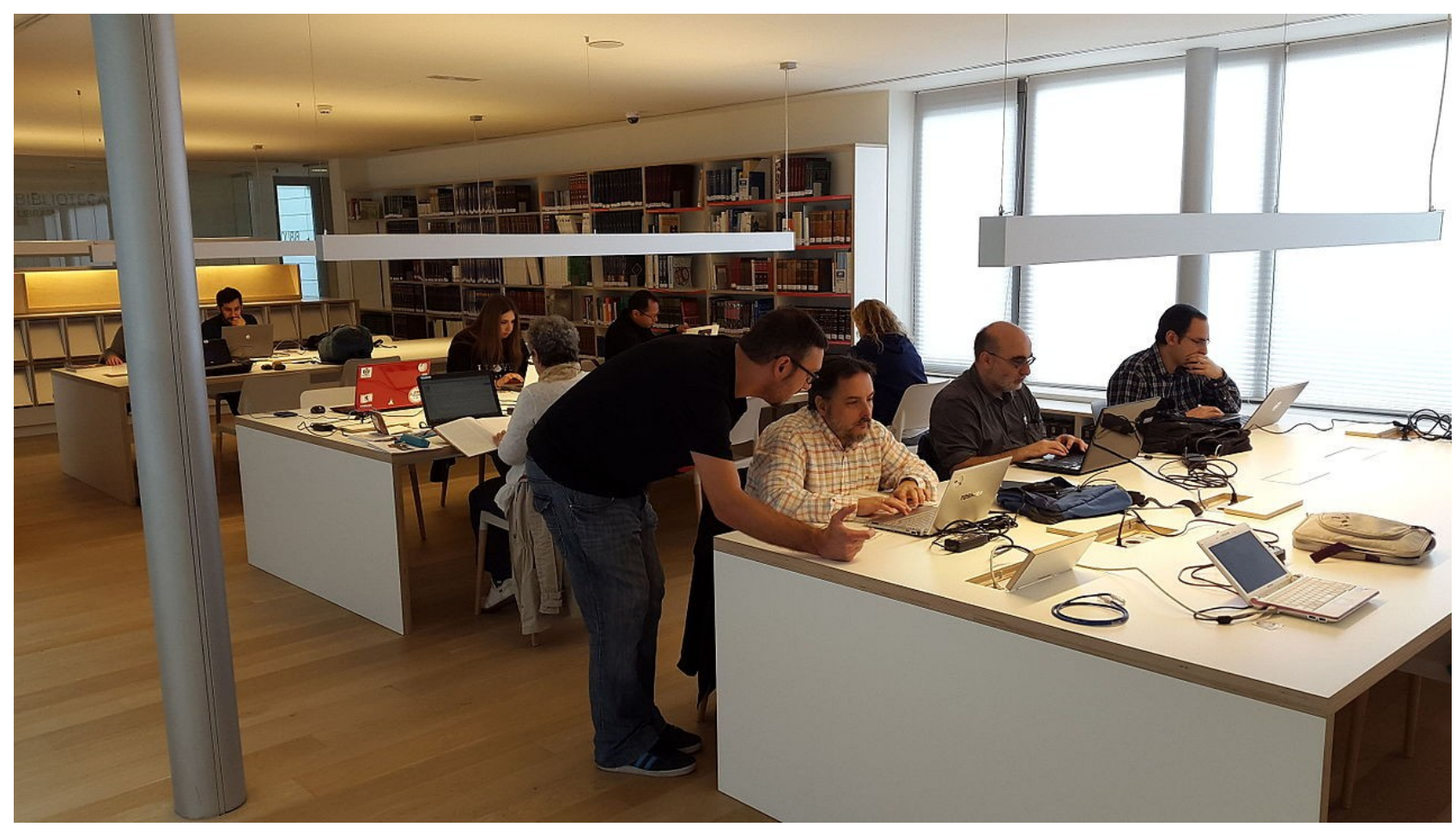

Ilustración 1: Trieditatón en el Museo Arqueológico Nacional (Fuente: Wikimedia Commons, https://commons.wikimedia.org/wiki/File:Trieditat\%C3\%B3n_Glaming_Madrid_06.jpg)

- Concurso de edición GLAMing Madrid Challenge: se trató de un concurso de edición, cuyo objetivo era favorecer la creación de contenidos sobre los tres museos, sus colecciones, y personalidades relacionadas con ellos, tanto en Wikipedia como en Wikidata ${ }^{24}$. Su duración fue de un mes, celebrándose entre el 14 de diciembre de 2015 y el 14 de enero de 2016. Para su desarrollo se crearon cuatro listas de artículos a crear o mejorar, correspondientes a cada uno de los museos y a mujeres célebres que hubieran estado vinculadas a cada museo. Para potenciar la participación se establecieron cinco premios, uno por cada museo, otro por la lista de mujeres y un último premio global. La coordinación, seguimiento y valoración del concurso fue llevada a cabo por Wikimedia España, mediante una página en Meta. Hubo treinta inscritos, de los cuales participaron activamente catorce, editando 335 artículos, 28 elementos de Wikidata y 10 páginas de categorías.

- Liberación de contenidos: la puesta a disposición de contenidos con licencias libres, procedentes de colecciones digitalizadas, es un objetivo fundamental de los proyectos GLAM. El resultado obtenido es doble, ya que por una parte difunden las colecciones de las instituciones participantes, y por otra se integran como ilustraciones en los diferentes contenidos de los proyectos Wikimedia. Los tres museos participantes liberaron un número limitado de fotografías de elementos pertenecientes a sus colecciones ${ }^{25}$, que inmediatamente comenzaron a ser usadas en artículos de Wikipedia en español y en otros idiomas. Sin embargo, las imágenes liberadas carecían de sus correspondientes conjuntos de metadatos, por lo que hubo que esperar a su preparación por los museos, tras lo cual las imágenes y sus metadatos fueron subidas a Wikimedia Commons usando una herramienta de software específicamente preparada a tal fin. 


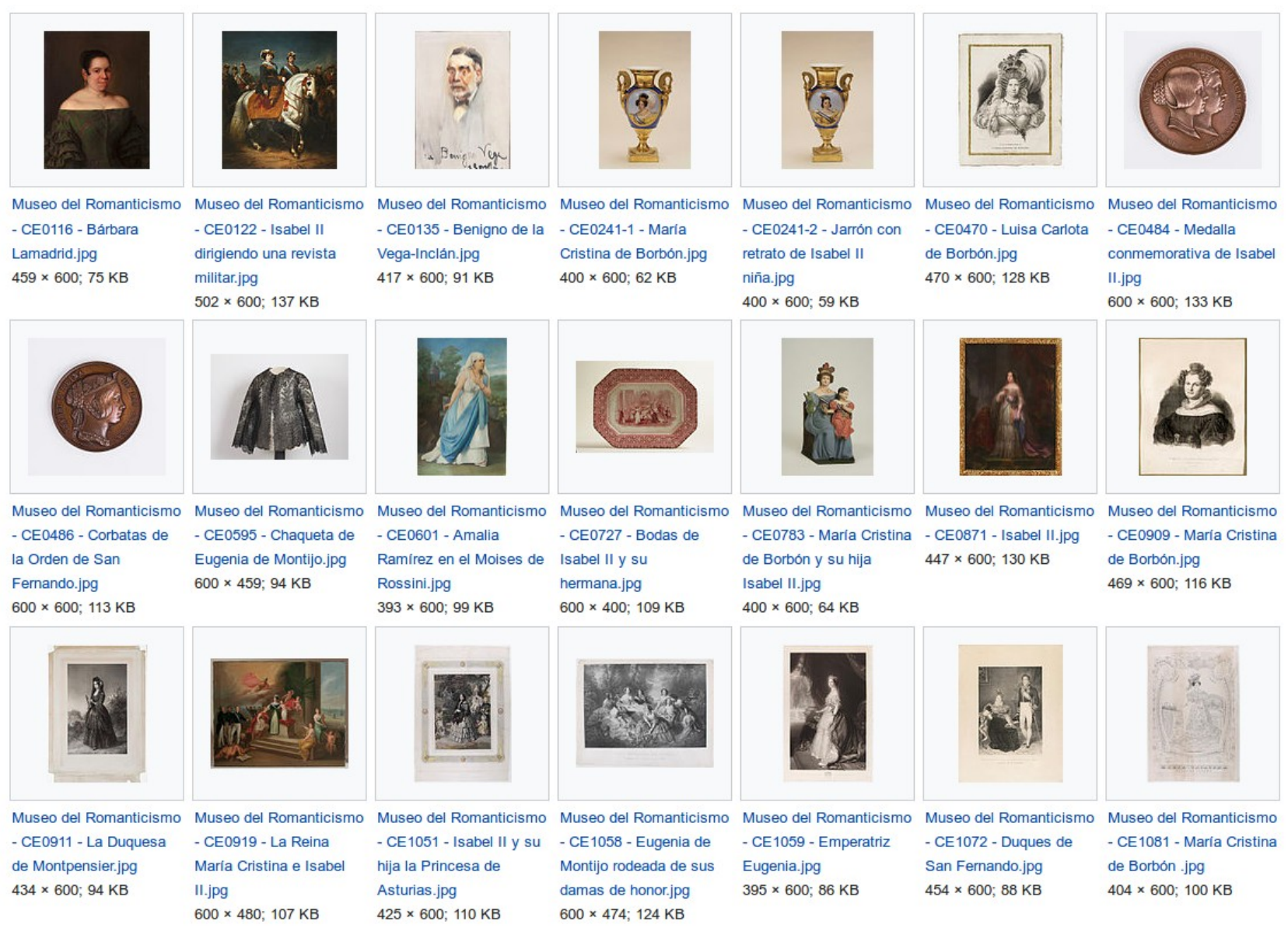

Ilustración 2: Material liberado por el Museo del Romanticismo en Wikimedia Commons (fuente: Wikimedia Commons, https://commons.wikimedia.org/wiki/Category:Files_provided_by_Museo_del_Romanticismo).

La difusión de resultados y la visibilidad que ésta podía aportar a los museos participantes se había establecido en el proyecto como un objetivo a cumplir. En primer lugar, se llevó a cabo una serie de acciones para visibilizar el proyecto dentro de la comunidad de los proyectos Wikipedia. Se creo una página específica en Wikipedia en español sobre el proyecto, que era actualizada regularmente, y la que se recogían la planificación, actividades y resultados del mismo. También se creó una categoría específica para los contenidos creados o aportados al mismo. Además del blog de Wikimedia España, que informaba periódicamente de las actividades del proyecto, el blog de la Wikimedia Foundation hizo referencia al proyecto en cuatro entradas, y la newsletter mensual This month in GLAM, que recoge las actividades GLAM llevadas a cabo en todo el mundo en relación con los proyectos Wikimedia se hizo eco de las acciones desarrolladas en cinco ocasiones. En lo que concierne a gabinetes y medios de prensa del estado español, las notas de prensa del proyecto y de sus actividades fueron objeto de una treintena de publicaciones en prensa, durante el período de ejecución, a las que habría que sumar varias más como consecuencia de la concesión del Premio Princesa de Asturias y de informaciones generales sobre la comunidad y los proyectos Wikimedia.

Finalmente, y como corolario del proyecto, Wikimedia España redactó y publico con licencia libre en 2016 un documento de trabajo titulado Introducción a los proyectos GLAM: cómo desarrollar una colaboración ${ }^{26}$, en el cual se recogen la experiencia adquirida durante el proyecto, las buenas prácticas adquiridas, y se señalan cuestiones y problemas a los que debe prestarse atención para obtener un resultado exitoso en este tipo de proyectos.

26 WIKIMEDIA ESPAÑA. Introducción a los proyectos GLAM: cómo desarrollar una colaboración. [en línea]. 2016. [consulta: 20 octubre 2017]. Disponble en: https://commons.wikimedia.org/wiki/File:C $\% \mathrm{C} 3 \% \mathrm{~B} 3 \mathrm{mo}$ _desarrollar_una_colaboraci\%C3\%B3n_GLAM.pdf 


\section{Resultados y buenas prácticas adquiridas.}

Los resultados obtenidos del proyecto han permitido disponer de información que hace posible delinear y planificar futuras actividades en entidades museísticas, de forma acorde con los contextos administrativo, social y cultural de las mismas. Las principales recomendaciones y buenas prácticas identificadas se resumen en los siguientes párrafos.

- Es necesario adaptar la planificación y las actividades a las características y contexto de cada institución. Hay que tener en consideración la estructura administrativa y de responsabilidades de la entidad que se trate.

- La planificación deberá incluir una clara y definida asignación de calendario, responsabilidades, necesidades y puntos de control para todas y cada una de las actividades contempladas.

- La formación previa del personal de los museos es un factor clave para el éxito de iniciativas GLAM. En el proyecto desarrollado se detectó el bajo nivel de conocimiento del personal sobre los aspectos técnicos y de gestión de Wikipedia y Wikimedia Commons.

- Es necesario explicar y enfocar claramente a las personas responsables de la entidad las características de las licencias libres, así como su impacto en la disponibilidad de materiales y colecciones.

- Hay que asegurar las condiciones materiales e infraestructura para la celebración de cursos y talleres, en cuanto influyen en la percepción de los usuarios de los mismos.

- La actividad de los bibliotecarios de preparación de materiales bibliográficos y documentales para la realización de acciones de edición se revela como indispensable.

- La combinación de actividades tipo taller, concurso y liberación de contenidos aumenta la calidad de los artículos editados.

- Es necesario establecer mecanismos de medida de resultados que permitan cuantificar el impacto de las acciones desarrolladas, de forma que pueda demostrarse su rentabilidad para la institución que las acoge.

- Hay que documentar los procesos desarrollados y los resultados obtenidos de manera sistemática, para disponer de prácticas replicables y de resultados comparables.

Wikimedia España llevó a cabo, además, dos encuestas sobre la experiencia de los participantes en las diferentes actividades llevadas a cabo. A tal fin se utilizó la herramienta Qualtrics, proporcionada por la Fundación Wikimedia. La primera de las encuestas fue respondida por el 68\% de los destinatarios. Entre otros datos, debe resaltarse que la mayor parte de los participantes en actividades eran mujeres, y que predominada un nivel educativo universitario. La franja de edad más numerosa era la contemplada entre los 31-40 y 51-50 años. La frecuencia de uso de Wikipedia predominante era de varias veces a la semana, en particular un uso diario, y la actividad casi exclusiva era la búsqueda y consulta de información de referencia. Debe destacarse que casi el 70\% de los participantes indicó que confiaba "bastante" en la calidad y el contenido de Wikipedia. En lo correspondiente a la edición de contenidos, alrededor del 75\% indicaba que no había editado nunca.

La segunda encuesta estaba orientada a conocer si los participantes había comenzado a desarrollar pautas de edición en Wikipedia como resultado de su participación en las actividades. En este caso el nivel de respuesta fue mucho más bajo, del $21 \%$ del total enviado. Dado el escaso número de respuestas, se realizó un análisis cualitativo de la información recibida. Las actividades de edición posterior fueron escasas, y se centraron en la creación o edición de contenido de artículos, la corrección gramática u ortográfica, o la inclusión de referencias bibliográficas. Como aspectos positivos resaltaron conocer los procesos de edición y de integración y uso de fotografías de Commons, así como la interacción con otros editores, mientras que valoraron negativamente el uso del código wiki y los parámetros de organización y redacción sintética de información. Los 
participantes resaltaron la importancia de disponer de monitores expertos durante los talleres, así como la conveniencia de darles una mayor duración temporal.

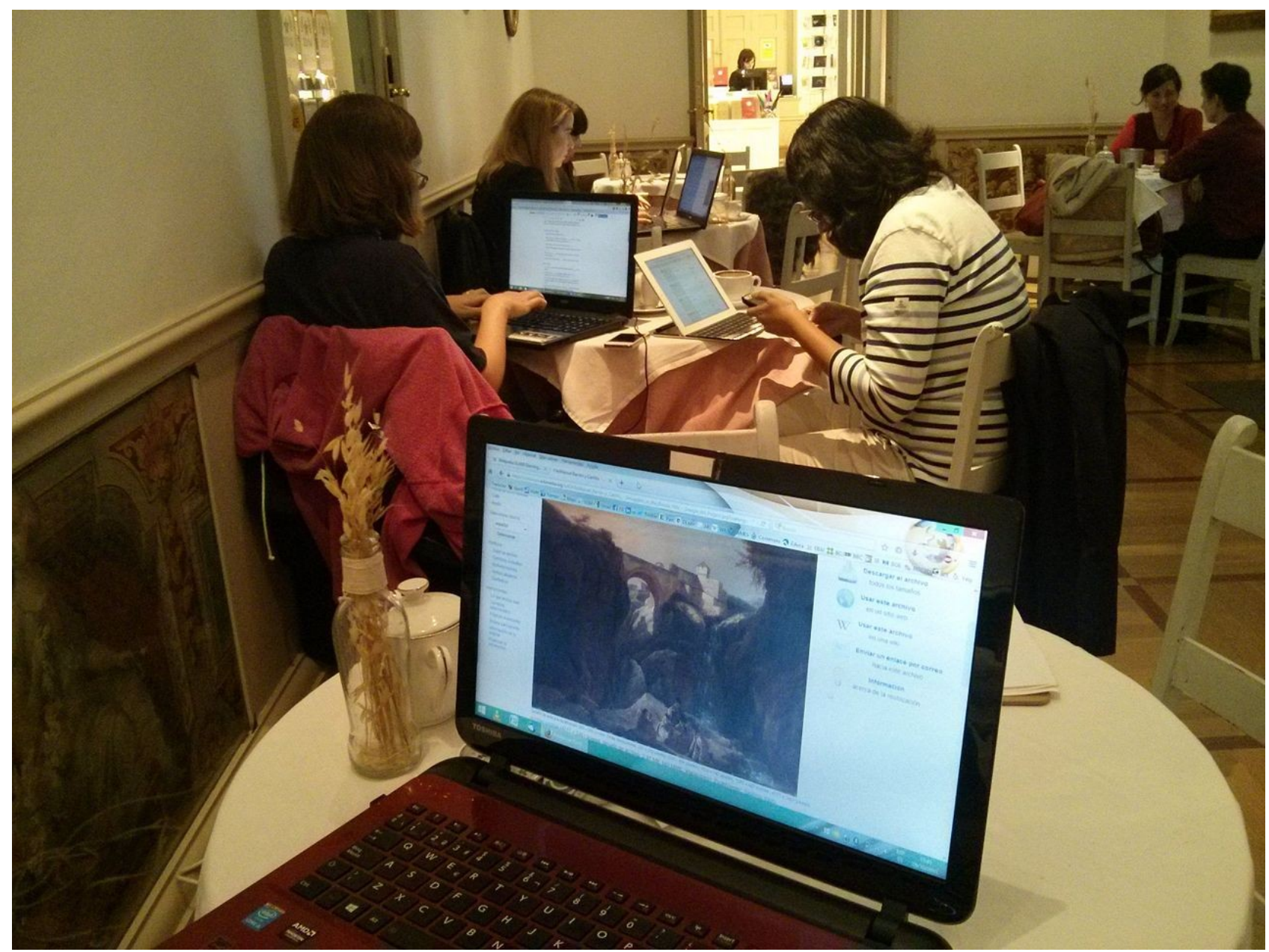

Ilustración 3: Taller en el Museo del Romanticismo (fuente: Wikimedia Commons, https://commons.wikimedia.org/wiki/File:Taller_01_Romanticismo_03.jpg)

\section{Conclusiones.}

El desarrollo y los resultados del proyecto GLAMing Madrid fueron exitosos, y demostraron la capacidad y potencial de la colaboracion entre instituciones museísticas y asociaciones, basadas en el trabajo voluntario y cooperativo, como Wikimedia España. Las múltiples posibillidades que se abren a los museos en el marco de proyectos OpenGLAM, y en particular en colaboración con Wikipedia y sus proyectos ya ha sido reseñada en otros trabajos ${ }^{27}$.

La publicación de material cultural digitalizado, con licencias que permiten el libre uso y reutilización, ofrece nuevas oportunidades para la investigación, el acceso y disfrute de la cultura, y la creación de nuevos productos y servicios de valor añadido. La incorporación al movimiento del acceso abierto facilitará el desarrollo de sistemas de recuperación y de agregación de contenidos. Para alcanzar los resultados deseables, las instituciones culturales necesitarán una decidida voluntad política, una adecuada planificación, el establecimiento y difusión de parámetros de medición de resultados, y la colaboración de otros actores implicados en iniciativas OpenGLAM en entornos de trabajo cooperativo.

27 TUNCHS, Tomas. Museum Documentation and Wikipedia.de: Possibilities, opportunities and advantages for scholars and museums. Museums and the Web 2007 [en línea]. 2007, [Consulta: 21 julio 2017]. Disponible en: http://www.museumsandtheweb.com/mw2007/papers/tunsch/tunsch.html. 


\section{Referencias bibliográficas}

AYERS, Phoebe. At scale, at large: Wikipedia \& Libraries. [en línea]. 2016. [consulta: 20 diciembre 2017]. Disponible en: https://medium.com/@phoebeayers/at-scale-at-large-wikipedia-libraries-3e90a9618398

BARTHOLMEI, Stephan, et alii. Opportunities for Academic and Research Libraries and Wikipedia. A Discussion Paper (Public Draft) Presented at the IFLA World Library and Information Congress 2016 in Columbus, Ohio. [en línea]. 2016. [consulta: 8 octubre 2017]. Disponible en: https://2016.ifla.org/wp-content/uploads/sites/2/2016/08/112IFLAWikipediaAcademicandResearchLibrariesDiscussioDRAFT.pdf

CATALANI, Luigi. Biblioteche e Wikimedia: strategie comuni per l'accesso aperto alla conoscenza e la costruzione collaborativa del sapere libero. JLIS.it, [en línea], 2017, vol. 8, núm. 3 [consulta: 22 noviembre 2017]. Disponible en: doi: $10.4403 /$ jlis.it- 12413

COMISIÓN EUROPEA. Recomendación de la Comisión de 27 de octubre de 2011 sobre la digitalización y accesibilidad en línea del material cultural y la conservación digital. [en línea]. Diario Oficial de la Unión Europea, 2011, L283/30-45 [consulta: 6 septiembre 2017]. Disponible en: http://eur-lex.europa.eu/LexUriServ/LexUriServ.do?uri=OJ:L:2011:283:0039:0045:ES:PDF

FENOLL, Carme, et alii. Opportunities for Public Libraries and Wikipedia. A Discussion Paper (Public Draft) Presented at the IFLA World Library and Information Congress 2016 in Columbus, Ohio. [en línea]. 2016. [consulta: 8 octubre 2017]. Disponible en: https://2016.ifla.org/wp-content/uploads/sites/2/2016/08/112IFLAWikipediaandPublicLibrariesDiscussionDRAFT.pdf

KAPSALIS, Effie. The Impact of Open Access on Galleries, Libraries, Museums, \& Archives. [en línea]. Smithsonian Institution, 2016, [consulta: 3 octubre 2017]. Disponible en: http://siarchives.si.edu/sites/default/files/pdfs/2016_03_10_OpenCollections_Public.pdf

OJEDA, Rubén. y HERNÁNDEZ-CAZORLA, Iván. Wikimedia España y la difusión del patrimonio cultural. Revista $P H$ [en línea]. 2017, vol. 91, págs. 36-37, [consulta: 2017-10-10]. Disponible en: http://www.iaph.es/revistaph/index.php/revistaph/article/view/3882

OJEDA, Rubén y WIKIMEDIA ESPAÑA. Proyecto GLAMing Madrid. [en línea]. 2016. [consulta: 7 septiembre 2017]. Disponible en: https://commons.wikimedia.org/wiki/File:Memoria_GLAMing_Madrid-es.pdf

ORLOWITZ, Jake y EARLEY, Patrick, Librarypedia: The future of Libraries and Wikipedia. [en línea]. TheDigitalShift, 2014. [consulta 2 de mayo de 2017]. Disponible en: http://www.thedigitalshift.com/2014/01/discovery/librarypedia-future-libraries-wikipedia/

RODRÍGUEZ-POSADA, Emilio José et alii. Wiki Loves Monuments 2011: experiencia en España y reflexiones desde la difusión del patrimonio cultural. Digithum, [en línea], 2012, n. 14, [consulta: 12 noviembre 2017] Disponible en: http://doi.org/10.7238/d.v0i14.1472

SAORÍN, Tomás. Iniciativas GLAM-Wiki: Wikipedia como oportunidad para instituciones culturales. Anuario ThinkEPI, [en línea], 2013. vol. 7, págs. 78-85. [consulta: 14 octubre 2017]. Disponible en: https://recyt.fecyt.es/index.php/ThinkEPI/article/view/30335

TUNCHS, Tomas. Museum Documentation and Wikipedia.de: Possibilities, opportunities and advantages for scholars and museums. Museums and the Web 2007 [en línea]. 2007, [Consulta: 21 julio 2017]. Disponible en: http://www.museumsandtheweb.com/mw2007/papers/tunsch/tunsch.html.

WIKIMEDIA ESPAÑA. Introducción a los proyectos GLAM: cómo desarrollar una colaboración. [en línea]. 2016. [consulta: 20 octubre 2017]. Disponble en: https://commons.wikimedia.org/wiki/File:C $\%$ C3\%B3mo_desarrollar_una_colaboraci\%C3\%B3n_GLAM.pdf 